\title{
Measuring Pulsatile Flow in Cerebral Arteries Using 4D Phase-Contrast MR Imaging
}

\author{
A. Wåhlin, K. Ambarki, R. Birgander, O. Wieben, K.M. Johnson, J. Malm, and A. Eklund
}

\section{ABSTRACT}

BACKGROUND AND PURPOSE: 4D PCMRI can be used to quantify pulsatile hemodynamics in multiple cerebral arteries. The aim of this study was to compare 4D PCMRI and 2D PCMRI for assessments of pulsatile hemodynamics in major cerebral arteries.

MATERIALS AND METHODS: We scanned the internal carotid artery, the anterior cerebral artery, the basilar artery, and the middle cerebral artery in 10 subjects with a single 4D and multiple 2D PCMRI acquisitions by use of a 3T system and a 32-channel head coil. We assessed the agreement regarding net flow and the volume of arterial pulsatility $(\Delta V)$ for all vessels.

RESULTS: 2D and 4D PCMRI produced highly correlated results, with $r=0.86$ and $r=0.95$ for $\Delta \mathrm{V}$ and net flow, respectively $(n=69$ vessels). These values increased to $r=0.93$ and $r=0.97$, respectively, during investigation of a subset of measurements with $<5 \%$ variation in heart rate between the 4D and 2D acquisition ( $n=31$ vessels). Significant differences were found for ICA and MCA net flow ( $P=.004$ and $P<.001$, respectively) and MCA $\triangle \mathrm{V}(P=.006)$. However, these differences were attenuated and no longer significant when the subset with stable heart rate ( $n=31$ vessels) was analyzed.

CONCLUSIONS: 4D PCMRI provides a powerful methodology to measure pulsatility of the larger cerebral arteries from a single acquisition. A large part of differences between measurements was attributed to physiologic variations. The results were consistent with 2D PCMRI.

ABBREVATIONS: $\mathrm{PCMRI}=$ phase-contrast MR imaging; $\mathrm{ACA}=$ anterior cerebral artery; $\Delta \mathrm{V}=$ volume of arterial pulsatility; $\mathrm{PC}$-VIPR = phase contrast with vastly undersampled isotropic projection reconstruction; $\triangle \mathrm{HR}=$ change in heart rate

$\mathbf{E}_{\mathrm{pec}}^{\mathrm{xac}}$ xcessive arterial pulsatility has been linked to poorer cognitive performance and brain atrophy among the elderly. ${ }^{1}$ Therefore, evaluation of this association is important for understanding pathophysiological processes related to cerebral aging and dementia. The pulsatility index, the resistive index, or a volume corresponding to vessel distention can be used to describe arterial pulsatility.

Received August 10, 2012; accepted after revision October 15.

From the Department of Radiation Sciences (A.W., K.A., R.B., A.E.), Center for Biomedical Engineering and Physics (K.A., A.E.), and Department of Clinical Neuroscience (J.M.), Umeå University, Umeå, Sweden; and Department of Medical Physics (O.W., K.M.J.) and Department of Biomedical Engineering (K.M.J.), University of Wisconsin, Madison, Wisconsin.

This project was supported by the Swedish Research Council, VINNOVA, and the Swedish Foundation for Strategic Research through their common initiative: "Biomedical Engineering for Improved Health"; Swedish Research Council Grant No. 621-2011-5216; EU, Objective two Norra Norland, CMTF; The County Council of Västerbotten; and the Swedish Heart and Lung Foundation.

Please address correspondence to Anders Wåhlin, PhD, Department of Radiation Sciences, Umeå University, SE-901 87, Umeå, Sweden; e-mail: Anders.Wahlin@ radfys.umu.se

-- Indicates open access to non-subscribers at www.ajnr.org

http://dx.doi.org/10.3174/ajnr.A3442
Whereas the indexes describe a mixture of vascular impedance, resistance, and compliance, ${ }^{2,3}$ the volume of arterial pulsatility represents the magnitude of accumulated downstream cyclic vessel distention, assuming a stationary downstream capillary flow, ${ }^{4,5}$ that is, the cyclic increase in arterial volume from the measurement level to the capillaries. Therefore, this measure is sensitive to the balance of factors such as downstream and upstream arterial compliance and cardiac stroke volume and thus provides a mechanically relevant alternative for analyzing vascular pulsatility. ${ }^{6}$

Traditionally, intracranial vascular pulsatility is estimated by use of Doppler sonography techniques ${ }^{1}$ or 2D PCMRI scans. ${ }^{4}$ These methods are associated with laborious workflows and rely on a trained neurovascular imager to make skillful interpretations and vessel localizations during the acquisition. 4D PCMRI allows a desirable separation of image acquisition and image interpretation because flow is collected over a large volume and subsequently analyzed off-line. ${ }^{7}$

Heavily accelerated 4D PCMRI makes it possible to assess arterial flow in the entire intracranial space while maintaining an acceptable scan time. ${ }^{8,9}$ This approach significantly eases the 
workflow, possibly reduces total scan time, and ensures the proper capture of all vessels in the prescribed imaging volume. Such 4D PCMRI techniques for intracranial measurements have been validated in vivo, ${ }^{10,11}$ but evaluations regarding pulsatile hemodynamics are lacking.

In the present study, we apply a radially undersampled $4 \mathrm{D}$ approach, PC-VIPR, ${ }^{12}$ to assess the volume of arterial pulsatility in major cerebral vessels. The main objective was to evaluate the agreement with conventional 2D PCMRI acquisitions.

\section{MATERIALS AND METHODS Subjects}

Ten healthy volunteers ( 7 male) were recruited (age [mean \pm SD], $37 \pm 9$ years; range, $29-53$ years). The local ethical review board approved the study, and written informed consent was obtained from all participants.

\section{MR Imaging}

All scans were performed by use of a clinical 3T scanner (Discovery MR 750; GE Healthcare, Milwaukee, Wisconsin) with a 32channel head coil. The imaging protocol started with a 3D timeof-flight acquisition, reconstructed in axial, sagittal, and coronal directions to provide vessel localization for the successive 2D PCMRI sequences. Second, 4D PCMRI data were acquired by use of a balanced 5-point ${ }^{13}$ PC-VIPR sequence, prescribed to cover the entire intracranial space. We used 16,000 radial projections; acquisition resolution, $300 \times 300 \times 300$; imaging volume, $22 \times$ $22 \times 22 \mathrm{~cm}$; reconstruction resolution, $320 \times 320 \times 320 \mathrm{~mm}$ (zero padded interpolation); velocity encoding, $110 \mathrm{~cm} \mathrm{~s}^{-1}$; TR/ TE, 6.5/2.7 ms; flip angle, $8^{\circ}$. Dynamic images were reconstructed by retrospective gating from the recorded peripheral pulse signal by use of temporal interpolation similar to view sharing in Cartesian acquisitions. ${ }^{14}$ Besides $3 \mathrm{D}$ velocity information for the 20 reconstructed time positions, a time-average composite complex difference image was reconstructed to provide anatomic detail of the vascular system. During the 9-minute 4D PCMRI acquisition, 5 2D PCMRI scans were planned by means of the time-of-flight images. The first measured left and right ICA and basilar artery at the level of the carotid C3-C4 segment ${ }^{15}$ (velocity encoding $80 \mathrm{~cm} \mathrm{~s}^{-1}$ ). The second and third 2D PCMRI sequences measured the M1 segment of left and right MCA respectively (velocity encoding, $110 \mathrm{~cm}$ $\mathrm{s}^{-1}$ ). The fourth and fifth 2D PCMRI sequences measured the A1 segment of left and right ACA, respectively (velocity encoding, $90 \mathrm{~cm}$ $\mathrm{s}^{-1}$ ). Other 2D PCMRI parameters were TR/TE, 7.6-8.5/4.1-5.0 ms; 3-mm section thickness; $15^{\circ}$ flip angle; in-plane resolution, $0.5 \times 0.5$ $\mathrm{mm} ; 6$ views per segment; and 2 averages. Thirty-two phases were reconstructed. Retrospective gating with peripheral sensor was used for all acquisitions.

Because the ICA has no branches close to the measurement location, this vessel segment provided the opportunity to investigate the internal consistency of the 4D PCMRI measurements. This consistency check was implemented by performing an additional segmentation, upstream and strictly separated (ie, no overlap) from the first segmentation, at carotid level C2-C3, in all ICAs. The separation distance between the 2 sections was approximately $1.5-2 \mathrm{~cm}$, in the direction along the ICA.

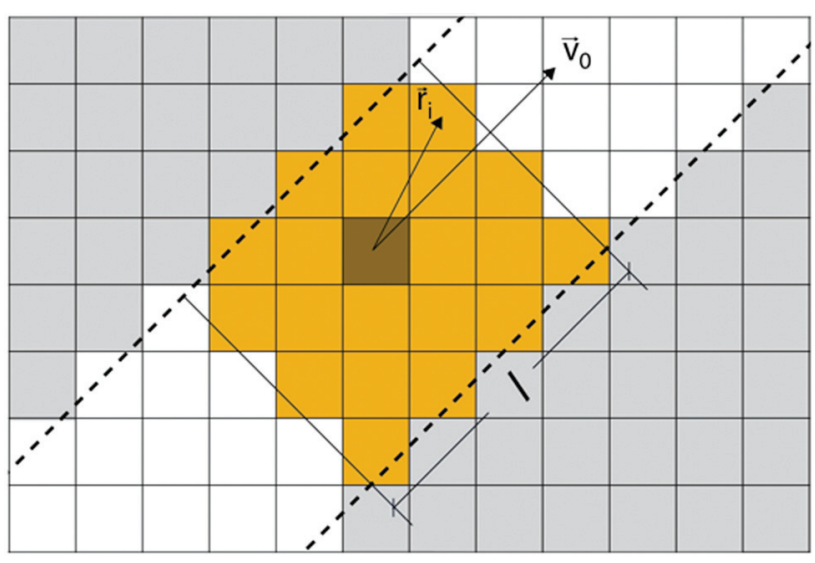

FIG 1. 2D representation of the segmentation procedure. Dashed lines represent the vessel lumen; yellow pixels are the segmentation result. White pixels represent pixel intensity of $\geq 18 \%$. Dark pixel is the user-defined reference pixel whose velocity direction was used to label a segment of length $l$. Segmentation was not updated between cardiac frames. Typical segmentations of the ICA, basilar artery, MCA, and ACA contained 230, 110,80, and 60 pixels, respectively.

\section{Segmentation}

A novel in-house-developed software was used for all 4D PCMRI segmentations. To segment a desired vessel in the 4D PCMRI, a reference pixel was selected by the user in a maximum intensity projection of the time-average composite complex difference image. A region-growing algorithm included neighboring connected pixels fulfilling 2 conditions: 1 ) a pixel value of $\geq 18 \%$ of the maximum time-averaged complex difference value (observed in the entire $320 \times 320 \times 320$ volume) and 2) the pixel was located within a desired section of the vessel.

The second condition was assessed by means of the relationship:

$$
\frac{\left|\bar{r}_{i} \cdot \bar{v}_{0}\right|}{\left\|\bar{v}_{0}\right\|} \leq \frac{l}{2}
$$

where $\bar{r}_{i}$ is a vector pointing from the reference pixel to the $i$ :th pixel, $\bar{v}_{0}$ is the vessel direction estimated by the velocity direction in the reference pixel, and $l$ is the desired segment length (Figs 1 and 2). The threshold of $18 \%$ was selected because a precalibration series indicated that this value provided complete vessel coverage without inclusion of neighboring static tissue. The segment length $l$ was selected as 5 pixel widths because this distance was similar to the section thickness of the 2D PCMRI scans.

At this processing stage, the flow for each time frame was calculated by use of the spatial average velocity (calculated from the average velocity vector within the segment) of the section multiplied by the area of the vessel, estimated from dividing the segment volume by $l$. Area overestimations originating from misalignment between $\bar{v}_{0}$ and the velocity direction of the complete segment were eliminated by use of a correction factor (cosine of misalignment between reference pixel direction and average direction of final segmentation). Interpolated velocity images, perpendicular to the vessel direction, were produced to ensure that no branching occurred within the section of interest. To investigate the reproducibility of the $4 \mathrm{D}$ segmentation procedure, the entire dataset was analyzed on 2 occasions. The results from the second occasion were only used in the reproducibility analysis. 


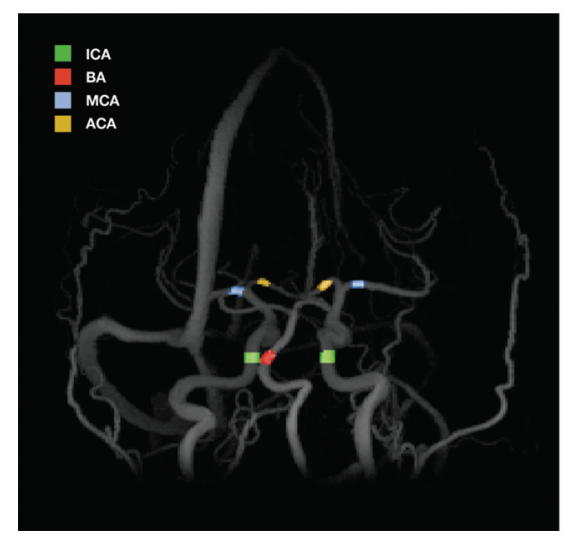

FIG 2. Maximum intensity projection image. Colored sections show segmentations produced for comparison with the 2D PCMRI data.

As a criterion standard reference, the 2D PCMRI data were analyzed by means of Segment v1.8 software (http://segment. heiberg.SE). The cross-sectional area of the artery was delineated manually by use of the magnitude images, and the size and position of the segmented area were kept constant during the cardiac cycle. The segmented area was drawn generously large to ensure proper capture of the complete vessel. In total, 7 vessels in 10 subjects were assessed, yielding a total of 70 vessels. However, 1 subject lacked a right A1 ACA segment (the right ACA was supplied by the left ACA through the anterior communicating artery). Therefore, all comparisons were based on 69 vessel segments.

\section{Net Flow and Pulsatility}

Net flow rates and the volume of $\Delta V$ were calculated by use of the same procedure for both 4D and 2D PCMRI waveforms. Net flow was derived as the temporal mean flow rate. On the basis of the assumption that capillary flow is nonpulsatile, the volume variation of the downstream arterial vessel distention, required to dampen the pulsatile arterial flow, can be estimated. This was performed by subtracting the net flow from the flow waveform and then calculating the cumulative integral over the remaining waveform (Fig 3). The difference between the maximum and minimum of this volume variation was defined as $\Delta \mathrm{V}^{4-6}$ and corresponds to the cyclic distention of downstream arteries required to convert the pulsatile arterial flow to a nonpulsatile capillary flow. Mathematically, this can be expressed as:

$$
\Delta V=\int_{\text {systole }}(q(t)-\bar{q}) d t
$$

where $q(t)$ is the flow rate and $\bar{q}$ is the net flow rate in the direction of the vessel. Here, systole is defined as the time of the cardiac cycle where the flow rate is higher than the net flow rate (ie, $q(t)>\bar{q}$ ).

\section{Statistical Analyses}

The agreement between 4D and 2D PCMRI was assessed by means of correlation and Bland-Altman plots. The paired-samples $t$ test was used to test systematic differences between the methods. To investigate the influence of physiologic variations,
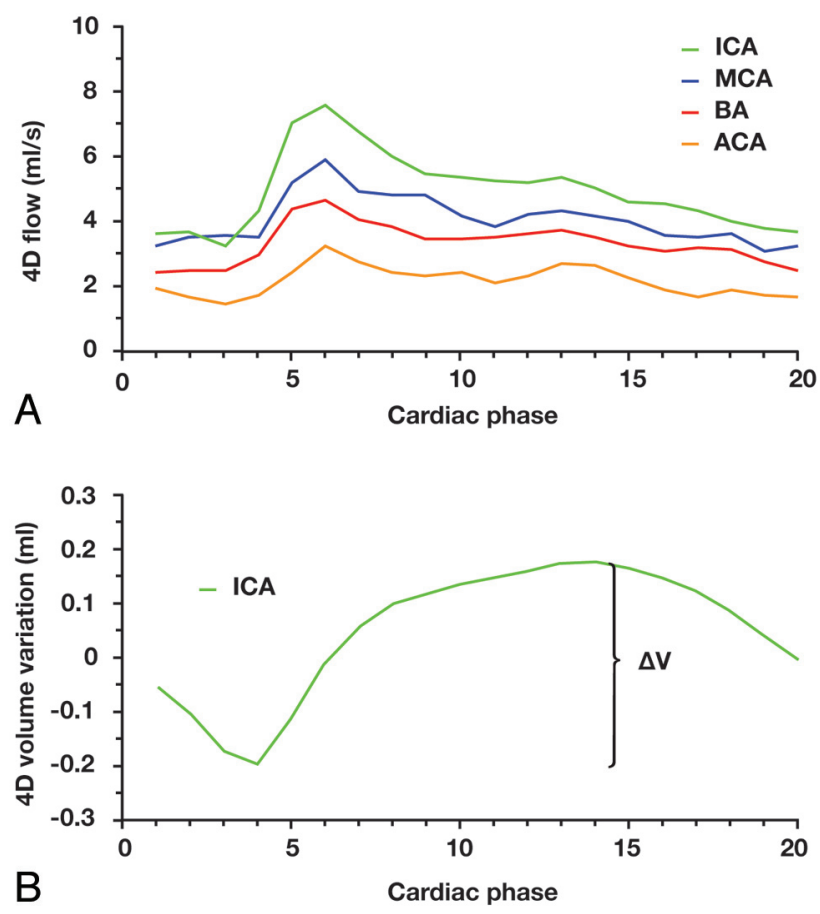

FIG 3. Arterial hemodynamics provided from a $4 D$ PCMRI quantification. A, Flow curves for vessels evaluated in this study. The right ICA, MCA, and ACA are shown. Cardiac phase 1-20 represents the time positions in the cardiac cycle of the reconstructed frames. B, ICA $\Delta \mathrm{V}$ calculation. Subtraction of net flow and calculation of the cumulative integral on the remaining waveform yield the cyclic volume variation of downstream arteries required to convert the pulsatile right ICA waveform to a nonpulsatile capillary flow. ICA $\Delta$ V was defined as the maximum minus minimum of this volume variation.

we also performed all analyses on a subset of measurements in which the difference in heart rate between the $4 \mathrm{D}$ and $2 \mathrm{D}$ acquisition $(\Delta \mathrm{HR})$ was $<5 \%(n=31)$. In the complete ensemble of measurements, the heart rate was 64 versus $63 \mathrm{bpm}$ for the $4 \mathrm{D}$ and 2D PCMRI acquisitions, respectively, with a standard deviation of the difference of $7 \mathrm{bpm}$. In the subset of measurements with $\Delta \mathrm{HR}$ $<5 \%$, heart rate was 68 versus $68 \mathrm{bpm}$ for the $4 \mathrm{D}$ and 2D PCMRI acquisitions, respectively, with a standard deviation of the difference of $2 \mathrm{bpm}$. The reproducibility of the $4 \mathrm{D}$ segmentation procedure was investigated by use of the coefficient of variation of the 2 repeated measurements.

\section{RESULTS}

Correlation plots for the net flow and $\Delta \mathrm{V}$ analysis are provided in Fig 4 . When analyzing all vessels simultaneously, the correlations were $r=0.86$ and $r=0.95$ for $\Delta \mathrm{V}$ and net flow, respectively ( $n=$ 69 vessels). These values increased to $r=0.93$ and $r=0.97$, respectively, when investigating only the subset with $\Delta \mathrm{HR}<5 \%$ ( $n=31$ vessels).

Corresponding Bland-Altman plots are shown in Fig 5. There were no significant statistical differences between $4 \mathrm{D}$ and 2D PCMRI-derived $\Delta \mathrm{V}$ or net flow when considering the entire ensemble of vessels ( $P=.11$ and $P=.98$, respectively). The relative 95\% limits of agreement were larger for $\Delta \mathrm{V}$ than for net flow. The repeated analyses on the subset of measurements with small differences in heart rate had greater influence on the limits of agreements for pulsatility than for net flow. 

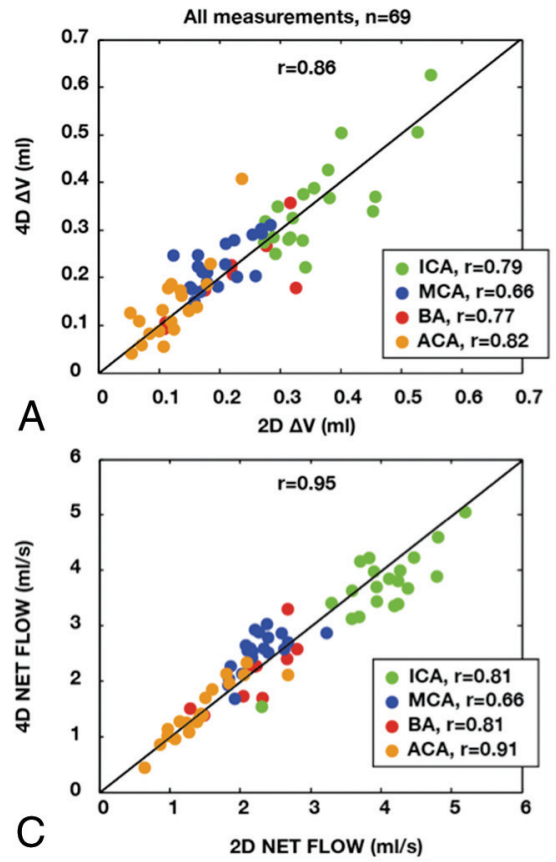
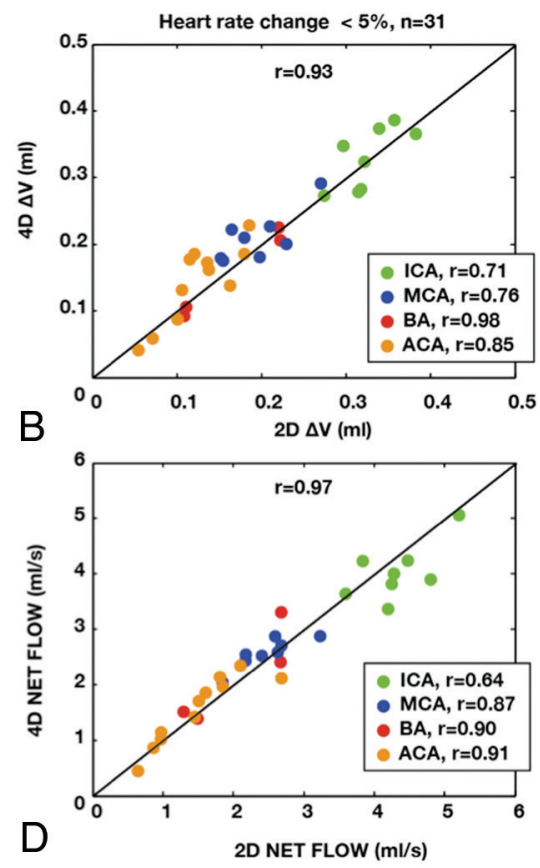

FIG 4. Correlation between $4 D$ and $2 D$ PCMRI quantifications of pulsatility and net flow. $A, \Delta V$ quantifications for all vessels. $B, \Delta \mathrm{V}$ quantifications, excluding measurements with large differences in heart rate. $C$, Net flow quantifications for all vessels. $D$, Net flow quantifications, excluding measurements with large differences in heart rate.
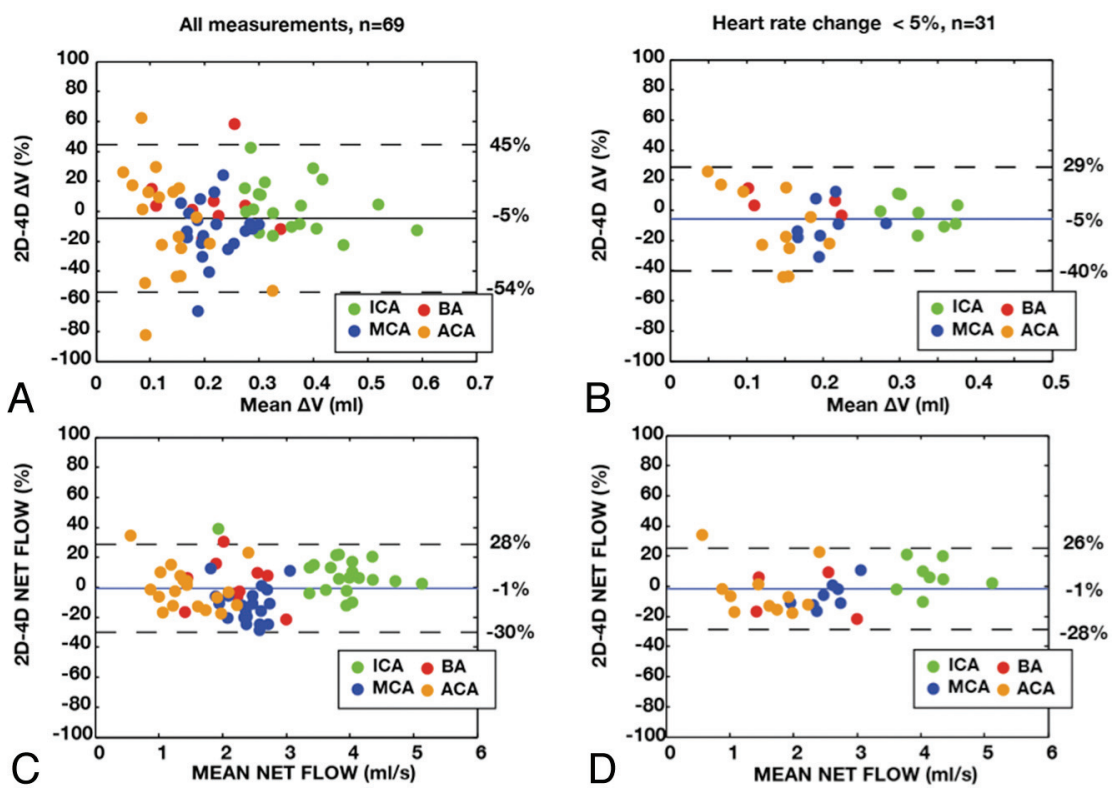

FIG 5. Agreement between $4 D$ and $2 D$ PCMRI quantifications of pulsatility and net flow. Solid lines represent average difference; dashed lines represent $95 \%$ limits of agreements. The relative difference was calculated as $(2 \mathrm{D}-4 \mathrm{D}) /([2 \mathrm{D}+4 \mathrm{D}] / 2)$. $A, \Delta \mathrm{V}$ quantifications for all vessels. $B, \Delta \mathrm{V}$ quantifications, excluding measurements with large differences in heart rate. $C$, Net flow quantifications for all vessels. $D$, Net flow quantifications, excluding measurements with large differences in heart rate.

Table 1 and Table 2 show mean values and differences for $\Delta \mathrm{V}$ and net flow, respectively. Significant differences appeared for the ICA and MCA when all measurements were taken into account in the analysis. These differences were attenuated when considering the stratified subset with $\Delta \mathrm{HR}$ $<5 \%$.

The coefficient of variation of the $4 \mathrm{D}$ segmentation procedure was $5 \%$ and $3 \%$ for $\Delta \mathrm{V}$ and net flow measurements, respectively. The internal consistency analyses along the ICA showed average differences of $0.000 \pm$ $0.034 \mathrm{~mL}$ (mean $\pm \mathrm{SD})$ and $0.090 \pm$ $0.230 \mathrm{~mL} / \mathrm{s}$ for ICA $\Delta \mathrm{V}$ and net flow, respectively. These differences were not statistically significant $(P=.83$ and $P=$ .10 , respectively, $n=20$ ). The correlation between the distal and proximal measurements were $r=0.94$ and $r=$ 0.94 for ICA $\Delta \mathrm{V}$ and net flow, respectively. The typical time required for all 2D PCMRI sequences, including the time-of-flight sequence for vessel localization, was 24 minutes. This was almost 3 times longer than the time required for the 4D PCMRI acquisition.

\section{DISCUSSION}

This study evaluated the use of 4D PCMRI for assessing pulsatile flow in large cerebral arteries. The combination of the PC-VIPR sequence and the proposed segmentation algorithm produced estimations of the volume of arterial pulsatility and net flow consistent with results obtained from $2 \mathrm{D}$ PCMRI. These results support the concept of the use of a single 4D PCVIPR acquisition to effectively assess pulsatility in many intracranial arteries, despite the disadvantage of the use of a single velocity sensitivity setting for all vessels instead of individually optimized sensitivity, as in the 2D PCMRI approach. The downside of the use of a single velocity sensitivity may be more significant in patients with stenotic arteries and a wider variation in flow velocities. For such investigations, a dual velocity encoding ${ }^{16}$ optimization may be warranted. Furthermore, future studies should evaluate 4D PCVIPR performance in more distal branches, for example, smaller arteries, as such measurements would be of great value when characterizing the cerebral collateral circulation.

There were no obvious qualitative differences between the $4 \mathrm{D}$ and $2 \mathrm{D}$ PCMRI images influencing the interpretation of the data. The 4D vessel segmentation algorithm was highly reproducible and did not require demanding calculations, for example, the waveforms appeared instantly as the user selected a reference pixel in the maximum intensity projection (Fig 2).

PC-VIPR net flow quantifications have previously been validated. Phantom measurements have demonstrated high accuracy 
Table 1: 4D and 2D PCMRI-based estimation of arterial pulsatility

\begin{tabular}{|c|c|c|c|c|c|c|}
\hline & Vessel Type & $n$ & $\begin{array}{c}\Delta V \text { 4D } \\
\text { Mean } \pm \mathrm{SD}(\mathrm{mL})\end{array}$ & $\begin{array}{c}\Delta V 2 D \\
\text { Mean } \pm \text { SD (mL) }\end{array}$ & $\begin{array}{c}\text { Difference } \\
\text { Mean } \pm \mathrm{SD}(\mathrm{mL})\end{array}$ & $P$ Value \\
\hline \multirow[t]{5}{*}{ All } & BA & 10 & $0.20 \pm 0.08$ & $0.21 \pm 0.08$ & $0.01 \pm 0.05$ & .504 \\
\hline & ICA & 20 & $0.35 \pm 0.10$ & $0.36 \pm 0.10$ & $0.01 \pm 0.06$ & .656 \\
\hline & $I_{C A}{ }^{a}$ & 20 & $0.35 \pm 0.10$ & & & \\
\hline & MCA & 20 & $0.23 \pm 0.05$ & $0.20 \pm 0.05$ & $-0.03 \pm 0.04$ & .006 \\
\hline & $\mathrm{ACA}$ & 19 & $0.14 \pm 0.08$ & $0.12 \pm 0.08$ & $-0.02 \pm 0.05$ & .102 \\
\hline$\Delta \mathrm{HR}$ & $\mathrm{BA}$ & 4 & $0.16 \pm 0.07$ & $0.17 \pm 0.07$ & $0.01 \pm 0.01$ & .268 \\
\hline \multirow[t]{3}{*}{$<5 \%$} & ICA & 8 & $0.33 \pm 0.05$ & $0.33 \pm 0.05$ & $-0.01 \pm 0.03$ & .668 \\
\hline & MCA & 8 & $0.21 \pm 0.04$ & $0.20 \pm 0.04$ & $-0.02 \pm 0.03$ & .116 \\
\hline & $\mathrm{ACA}$ & 11 & $0.14 \pm 0.06$ & $0.13 \pm 0.06$ & $-0.02 \pm 0.03$ & .074 \\
\hline
\end{tabular}

Note:-BA indicates basilar artery; ICA, internal carotid artery; MCA, middle cerebral artery; ACA, anterior cerebral artery; $\Delta \mathrm{HR}$, difference in heart rate; $\Delta \mathrm{V}$, volume of arterial pulsatility.

${ }^{a}$ Indicates proximal ICA measurement for internal consistency analyses.

Table 2: 4D and 2D PCMRI-based estimations on arterial net flow

\begin{tabular}{|c|c|c|c|c|c|c|}
\hline & Vessel Type & $n$ & $\begin{array}{c}\text { Net Flow 4D } \\
\text { Mean } \pm \text { SD } \\
(\mathrm{mL} / \mathrm{s})\end{array}$ & $\begin{array}{c}\text { Net Flow 2D } \\
\text { Mean } \pm \text { SD } \\
(\mathrm{mL} / \mathrm{s})\end{array}$ & $\begin{array}{c}\text { Difference } \\
\text { Mean } \pm \text { SD } \\
(\mathrm{mL} / \mathrm{s})\end{array}$ & $P$ Value \\
\hline \multirow[t]{5}{*}{ All } & BA & 10 & $2.15 \pm 0.58$ & $2.18 \pm 0.49$ & $0.03 \pm 0.34$ & .787 \\
\hline & ICA & 20 & $3.72 \pm 0.70$ & $4.02 \pm 0.62$ & $0.29 \pm 0.40$ & .004 \\
\hline & $\mathrm{ICA}^{\mathrm{a}}$ & 20 & $3.81 \pm 0.71$ & & & \\
\hline & MCA & 20 & $2.54 \pm 0.36$ & $2.26 \pm 0.34$ & $-0.28 \pm 0.29$ & $<.001$ \\
\hline & $\mathrm{ACA}$ & 19 & $1.47 \pm 0.52$ & $1.44 \pm 0.49$ & $-0.02 \pm 0.21$ & .627 \\
\hline$\Delta \mathrm{HR}$ & BA & 4 & $2.16 \pm 0.89$ & $2.02 \pm 0.74$ & $-0.13 \pm 0.39$ & .538 \\
\hline \multirow[t]{3}{*}{$<5 \%$} & $\mathrm{ICA}$ & 8 & $4.04 \pm 0.51$ & $4.32 \pm 0.51$ & $0.28 \pm 0.43$ & .108 \\
\hline & MCA & 8 & $2.58 \pm 0.27$ & $2.46 \pm 0.42$ & $-0.12 \pm 0.23$ & .173 \\
\hline & ACA & 11 & $1.55 \pm 0.61$ & $1.49 \pm 0.61$ & $-0.06 \pm 0.26$ & .427 \\
\hline
\end{tabular}

Note:-BA indicates basilar artery; ICA, internal carotid artery; MCA, middle cerebral artery; ACA, anterior cerebral artery; $\Delta \mathrm{HR}$, difference in heart rate; $\Delta \mathrm{V}$, volume of arterial pulsatility.

${ }^{a}$ Indicates proximal ICA measurement for internal consistency analyses.

The 4D PCMRI ICA internal consistency analyses demonstrated an internal variance (squared standard deviation of the difference between the 2 measurements) explaining approximately onethird of the variance of differences found between 4D and 2D PCMRI acquisitions, both for $\Delta \mathrm{V}$ and net flow. The remaining two-thirds of variance found between 2D and 4D PCMRI for the ICA measurements probably represented a mixture of 2D PCMRI internal variance and physiologic fluctuations caused by the separation in time between the 2 acquisitions.

There are several factors that potentially generate differences between $4 \mathrm{D}$ and 2D PCMRI measurements. First, the 4D PCMRI acquisitions were significantly longer than a single $2 \mathrm{D}$ scan. If the time of peak flow and flow amplitude are unstable between heartbeats, this could generate a more pronounced temporal smoothing in the $4 \mathrm{D}$ scan because the reconstructed cardiac cycle represents an average of all acquired heartbeats. Additionally, the resolution differs between the 2 methods. Potentially, this causes some degree of partial volume effects, normally displayed as a flow overestimation in vessels with a

of the PC-VIPR sequence, ${ }^{8}$ and results agree with net flow and average velocity measurements by $2 \mathrm{D}$ PCMRI $^{8,11}$ and transcranial Doppler. ${ }^{11}$ For peak systolic and diastolic velocities, previous investigations have displayed a $10 \%$ underestimation and a $9 \%$ overestimation, respectively, compared with 2D PCMRI. ${ }^{11}$ Probably, this is an effect from different temporal resolutions. Given the agreement between 4D and 2D PCMRI found in the present study, we hypothesize that $\Delta \mathrm{V}$ calculations are less influenced by differences in temporal resolution.

In the present study, arterial pulsatility measurements appeared more challenging (ie, wider limits of agreement) compared with net flow measurements. However, the limits of agreement for arterial pulsatility were narrowed by approximately onethird when calculated from measurements with a small difference in recorded heart rate. These data indicate that a large part of the variation between the 2 measurements was a result of physiologic variations, in agreement with previous data. ${ }^{5}$

The standard deviation of the difference between $2 \mathrm{D}$ and $4 \mathrm{D}$ PCMRI for ICA $\Delta \mathrm{V}$ and net flow was $0.06 \mathrm{~mL}$ and $0.40 \mathrm{~mL} / \mathrm{s}$, respectively. A previous study conducted with the use of repeated 2D PCMRI acquisitions showed intrasubject standard deviations of $0.067-0.070 \mathrm{~mL}$ and $0.32-0.33 \mathrm{~mL}$ for ICA $\Delta \mathrm{V}$ and net flow, respectively. ${ }^{5}$ This indicated that $2 \mathrm{D}$ and $4 \mathrm{D}$ PCMRI were associated with the same level of measurement variability, supporting that at least for the ICA, 4D PCMRI can replace 2D PCMRI without compromising measurement performance. small diameter/pixel size ratio. ${ }^{5}$ In the present study, significant differences between 4D and 2D PCMRI for ICA and MCA flows disappeared when eliminating measurements with large differences in heart rate, but a tendency of 4D PCMRI to underestimate ICA and overestimate MCA flows persisted. However, even if this represented actual offsets, the magnitudes were smaller than $10 \%$, whereby for most applications this will not constitute a limiting factor.

It should be noted that the flow calculation method of the $4 \mathrm{D}$ data assumes that the vessel is straight and not curved within the measurement section. This mimics requirements associated with 2D PCMRI acquisitions. In this study, we ensured that flow was captured in straight vessel sections, without branches. Future studies focusing on flow in more complex-shaped arteries may benefit from more sophisticated procedures to characterize flow in the $4 \mathrm{D}$ data.

\section{CONCLUSIONS}

4D PCMRI provided a practical methodology to estimate pulsatility of large cerebral arteries. The results were consistent with $2 \mathrm{D}$ PCMRI estimations. The simple workflow requires minimal prospective planning, and the output allows comprehensive analysis of pulsatile intracranial hemodynamics. Therefore, 4D PCMRI is an important tool for future investigations regarding altered intracranial vascular pulsatility. 
Disclosures: Anders Wåhlin—RELATED: Grant: Research grants, ${ }^{*}$ Comments: This project was supported by the Swedish Research Council, VINNOVA, and the Swedish Foundation for Strategic Research through their common initiative: 'Biomedical Engineering for Improved Health'; Swedish research council Grant No. 621-2011-5216; EU, Objective two Norra Norland, CMTF; The County Council of Västerbotten; and the Swedish Heart and Lung Foundation. Oliver Wieben—UNRELATED: Other: GE Healthcare, ${ }^{\star}$ Comments: My department receives research support from GE Healthcare. Kevin Johnson—OTHER RELATIONSHIPS: UW Madison receives research support from GE Healthcare. Jan Malm—RELATED: Grant: Swedish Heart and Lung Foundation; UNRELATED: Royalties: Likvor AB. Anders Eklund—RELATED: Grant: Swedish Research Council;* UNRELATED: Payment for Lectures (including service on speakers bureaus): DePuy International Limited; Royalties: Likvor AB (*money paid to institution).

\section{REFERENCES}

1. Mitchell GF, van Buchem MA, Sigurdsson S, et al. Arterial stiffness, pressure and flow pulsatility and brain structure and function: the Age, Gene/Environment Susceptibility-Reykjavik study. Brain 2011;134:3398-407

2. Michel E, Zernikow B. Gosling's Doppler pulsatility index revisited. Ultrasound Med Biol 1998;24:597-99

3. Bude RO, Rubin JM. Relationship between the resistive index and vascular compliance and resistance. Radiology 1999;211:411-17

4. Wåhlin A, Ambarki K, Birgander R, et al. Assessment of craniospinal pressure-volume indices. AJNR Am J Neuroradiol 2010;31:1645-50

5. Wåhlin A, Ambarki K, Hauksson J, et al. Phase contrast MRI quantification of pulsatile volumes of brain arteries, veins, and cerebrospinal fluids compartments: repeatability and physiological interactions. J Magn Reson Imaging 2012;35:1055-62

6. Bateman GA, Levi CR, Schofield P, et al. The venous manifestations of pulse wave encephalopathy: Windkessel dysfunction in normal aging and senile dementia. Neuroradiology 2008;50:491-97

7. Hsiao A, Lustig M, Alley MT, et al. Rapid pediatric cardiac assessment of flow and ventricular volume with compressed sensing par- allel imaging volumetric cine phase-contrast MRI. Am J Roentgenol 2012;198:W250-59

8. Gu T, Korosec FR, Block WF, et al. PC VIPR: a high-speed 3D phasecontrast method for flow quantification and high-resolution angiography. AJNR Am J Neuroradiol 2005;26:743-49

9. Hope MD, Purcell DD, Hope TA, et al. Complete intracranial arterial and venous blood flow evaluation with 4D flow MR imaging. AJNR Am J Neuroradiol 2008;30:362-66

10. Wu Y, Chang W, Johnson KM, et al. Fast whole-brain 4D contrastenhanced MR angiography with velocity encoding using undersampled radial acquisition and highly constrained projection reconstruction: image-quality assessment in volunteer subjects. AJNR Am J Neuroradiol 2011;32:E47-50

11. Chang W, Landgraf B, Johnson KM, et al. Velocity measurements in the middle cerebral arteries of healthy volunteers using 3D radial phase-contrast HYPRFlow: comparison with transcranial Doppler sonography and 2D phase-contrast MR imaging. AJNR Am J Neuroradiol 2011;32:54-59

12. Frydrychowicz A, Landgraf B, Wieben O, et al. Scimitar syndrome: added value by isotropic flow-sensitive four-dimensional magnetic resonance imaging with PC-VIPR (phase-contrast vastly undersampled isotropic projection reconstruction). Circulation 2010;121:e434-36

13. Johnson KM, Markl M. Improved SNR in phase contrast velocimetry with five-point balanced flow encoding. Magn Reson Med 2010;63:349-55

14. Liu J, Redmond MJ, Brodsky EK, et al. Generation and visualization of four-dimensional MR angiography data using an undersampled 3-D projection trajectory. IEEE Trans Med Imaging 2006;25:148-57

15. Bouthillier A, van Loveren HR, Keller JT. Segments of the internal carotid artery: a new classification. Neurosurgery 1996;38:425-33

16. Nett EJ, Johnson KM, Frydrychowicz, et al. Four-dimensional phase contrast MRI with accelerated dual velocity encoding. J Magn Reson Imaging 2012;35:1462-71 